\title{
The technology research of EPC production system
}

\author{
Tao-ping YAN \\ Faculty of Transportation Engineering \\ HuaiYin Institute of Technology \\ Huai'an, China \\ e-mail: ytp709803816@sohu.com
}

\section{Keywords: EPC; Casting production ;Impregnation technology;research}

Abstract: EPC process, based on the casting structure, technical requirements, production volume, production conditions determines the casting solution, process planning. In this paper, the author from three aspects, the analysis of die casting process design principles, design principles and infiltration technology, makes a deeper understanding on the EPC process, and put forward some standards and requirements of the EPC process technology, which avoid the casting defects and waste caused by technical factors.

\section{Introduction}

EPC (also called Lost Foam Casting) is similar to the size and shape of paraffin or foam model of bond portfolio into the cluster model, brushing coating and drying, buried in the dry quartz vibration model, pouring under vacuum, and is the model for gasification, which occupies the position of liquid metal model, casting foundry method the formation of solidification cooling. Lost foam casting is a new process of near zero allowance and precision molding. The process does not need modulo, parting, and sand core. Thus the cast has no flash, burr and draft, and reduces the dimension error caused by core portfolio. The casting surface roughness can reach Ra3.2 to $12.5 \mu$ $\mathrm{m}$. The casting dimension accuracy is up to IT7 to 9. The machining allowance for a maximum is up 1.5 to $2 \mathrm{~mm}$. Compared with traditional sand casting method, they can greatly reduce the machining cost, and the mechanical processing $40 \%$ to 50\%. For EPC has certain specifications and requirements, this paper mainly analyses the EPC process design principles with infiltration technique to determine the casting solutions and solve the problems of the lost foam casting tissue shrinkage, poor air tightness of EPC casting.

\section{The design discipline of Lost foam casting}

In addition to follow the general principles of casting design, lost foam casting have its own characteristics in the design. In order to guarantee the precision and rigidity of white mold, the minimum wall thickness should be greater than the minimum wall casting alloy filling thickness. At the same time, the section of cast on general is larger than three bead diameter. To ensure the module stiffness and reduce the deformation modeling, process bars must be increased in the inner die. Process bars should be set with symmetrical structure which play a role in the state of compression when in shape, the pouring in the final filling part ,the filling up slag and rigid fixation in solidification ${ }^{[1]}$. 


\section{The design discipline of lost foam casting process engineering}

Expendable pattern casting process design principle determines the casting solution and process planning according to the structural characteristics, technical requirements, production volume, production conditions. The keys include rates, types of white mold surface and the design of gating system contraction.

\section{Shrinkage}

Because of alloy shrinkage, the casting size after cooling will be slightly smaller than the sample size. To make sure the proper size, casting model size must be bigger than the ratio of one alloy casting shrinkage. Complex degree, casting size and structure shrinkage allowance usually have connections with alloy casting shrinkage rate, often with shrinkage said.

In view of the differences of casting shrinkage anisotropy, small size or low precision casting using a mold rate of contraction will not bring about problems. But the position of high precision with thin wall and large castings will be influenced. So before making the formal casts, we often have the necessary cutting by hand line to measure actual shrinkage in casting rate anisotropy and make the expensive die size correct.

\section{Principle of the determination of die.it parting surface}

The parting surface should be flat as much as possible so as to facilitate the module assembly. Besides, we should put machining reference casting processing surface or most of the processing surface, the processing datum on the same die to ensure the accuracy of the castings as far as possible.

Under the conditions of meeting the mass process production, the less die is, the better it will be. This mainly because more dies will increase tooling accessories which makes high research cost and long research period on one hand. On the other hand, the production process is complicated. The model geometry precision is difficult to control. And module assembly is too large. It will produce large amount of gas when pouring, which deteriorate the environment and make porosity of castings easily ${ }^{[2]}$.

\section{The Determining principle of pouring system}

Lost foam casting pouring system is completely different from hollow casting. Lost foam casting carries on mainly according to the related theory, without considering the disappearance of special casting process. It directly affects the disappearance of application development technology casting. The main function of pouring system is ensuring the EPC module stiffness, out of control pattern of pyrolysis and die pyrolysis, letting the molten metal into the mould of rational distribution. And it helps avoid the turbulence of molten metal and the bus production, ensure the filling liquid metal which has a reasonable temperature distribution in the mold. The followings are general principles of the design of the gating system in EPC listed from the angel of filling and touch kind pyrolysis products:

The pouring system of EPC must be convenient for module assembly that ensure the module stiffness and strength in shape.

Each unit should use the geometry of the gating system of low modulus, and adopt a hollow structure as far as possible to reduce the number of pyrolysis products and the exclusion of convenient foam pyrolysis product. Sprue height influence on the filling ability is smaller, while ensuring the casting compaction eat sand amount and minimizing the sprue height. Charge ability, filling time, filling velocity and filling metal front shape have little relations with tongue size of ingate dimension. Some of the tongue size of ingate dimension should be as large as possible to ensure module stiffness ${ }^{[3]}$. 


\section{The impregnation technology of lost foam casting}

In order to solve the problem of lost foam casting, casting shrinkage and poor air tightness, the most common technique is the infiltration process, namely the plugging. The so-called "infiltration", is that under certain conditions the infiltration agent infiltrated micropore castings. After curing the infiltration pore and pore wall filler in casting integrated block pores, process technology, the parts can meet the pressure, seepage and leakage condition. In recent years, with the rapid development of infiltration processing equipment and materials, the advanced industrial countries have called for dealing with the pressure casting with the infiltration process. And It becomes the production process recognized subsequent processing casting process. The infiltration process is divided into local infiltration and infiltration in two categories ${ }^{[4]}$.

\section{Infiltration agent}

Infiltration agent is a liquid substance composed of inorganic or organic compounds prepared by casting, which is used to seal the micro defects. The requirements of infiltration agent performance is good permeability and adhesion of casting defects. After infiltration agent curing, it should form a solid whole and casting. Curing material has good solvent resistance and mechanical properties, and is close to the body at the same time. Infiltration agent should also be non-toxic, no pollution, water gas, good stability, safety and reliability, is easy to be storaged and low cost and etc. Infiltration agent can be divided into two categories and organic.

Inorganic infiltration agent is in silicate type. Its main component is sodium silicate (commonly known as water glass), adding proper amount of inorganic metal salts, stabilizing agent, curing agent, metal oxides and toughening agent. Sodium silicate, the earliest use of infiltration agent, is widely used in the forty or fifty's of the twentieth Century. The biggest feature is $5001{ }^{\circ} \mathrm{C}$ high temperature capability, and wide material source, low cost. Its disadvantages are the high viscosity, wettability. And after dehydration, its shrinkage rate is very high and the production efficiency is low. After dried, the material brittle, easy to fall off, and the leakage rate is higher. The impregnation impregnation effect is good for aluminum castings with bigger pore defect. But for the pore tiny pore density large castings, the sealing effect is not good. The surface is still possible leakage. It takes a longer time to cure in the natural temperature. Infiltration casting should be placed at room temperature $24 \mathrm{~h}$ before pressure test. It is not suitable for large batch of castings ${ }^{[5]}$.

\section{Vacuum pressure impregnation process}

The most widely used method to produce castings in the factory at home or abroad, is vacuum pressure infiltration. The domestic development of LJS system of vacuum pressure impregnation did not completely improved. In the overall design and the degree of automation, it is close to the level of similar products in the world. Vacuum pressure impregnation process mainly goes on by shift process sequence which can be divided into pre treatment, infiltration and after processing the 3 stages in the vacuum pressure tank. Pre treatment consists of the casting degreasing, cleaning, and drying alternate. The main objective is to remove the oil, parts of the surface of the metal debris and dust, increase the impregnation quality, prevent pollution and mechanical impurities into the infiltration of liquid penetration and cohesion. The commonly used solvent degreasing ways contain degreasing method, alkali degreasing degreasing and electrochemical. In order to improve the penetration effect, it is necessary to cast in the degreasing cleaning, remove the water rinse, and then dry the cast at $8090{ }^{\circ} \mathrm{C}$. The drying after washing is very important so as to prevent large amounts of water into the vacuum pump ${ }^{[6]}$. 


\section{Conclusion}

To sum up, lost foam casting has its own characteristics, compared with other casting process. The key to the success of casting production is grasping the EPC process design principle. To master the infiltration technique can effectively prevent the defects of castings, and improve the quality of castings.

\section{Reference}

[1]Maosheng Yang; Bao yin; Jinyuan Ma etc. EPC production system design casting and its application----- Foundry equipment research;2001(4):1-5

[2]Na Teng; Shantang Cao etc. Foundry and design manual factory. Beijing. Machinery Industry Press;2010

[3] Wenhao He. Raw performance analysis and its application in ductile iron casting[D]. Huazhong University of Science and Technology.2010

[4]Chunxi Zhu; Xiaojia Qiu; JingHan; Study of Cockle Defect in EPC Castings [J]. Journal of Luoyang Technology College .2006(3):16-18

[5] Guangze Liang; Full mold casting [M]; Shanghai Science and Technology Press. 1986

[6] Qi'ai Fu; Deshun Yu; Vacuum full mould casting [M]. New times press; 1991. 